\title{
Dynamical Breakup of the Fermi Surface in a doped Mott Insulator
}

\author{
M. Civelli ${ }^{1}$, M. Capone ${ }^{2}{ }^{3}$, S. S. Kancharla ${ }^{4}$, O. Parcollet ${ }^{5}$ and G. Kotliar ${ }^{1}$ \\ 1 Physics Department and Center for Materials Theory, Rutgers University, Piscataway NJ USA \\ 2 INFM-SMC and Istituto dei Sistemi Complessi CNR, Via dei Taurini 19, I-00185, Rome, Italy \\ 3 Physics Department, University of Rome "La Sapienza", Piazzale A. Moro 5, I-00185, Rome, Italy \\ 4 Departement de physique and Regroupement quebecois sur les materiaux de pointe, \\ Université de Sherbrooke, Sherbrooke, Quebec J1K 2R1, Canada and \\ 5 Service de Physique Theorique, CEA Saclay , 91191, Gif-Sur Yvette, France
}

(Dated: September 26, 2018)

\begin{abstract}
The evolution from an anomalous metallic phase to a Mott insulator within the two-dimensional Hubbard model is investigated by means of the Cellular Dynamical Mean-Field Theory. We show that the density-driven Mott metal-insulator transition is approached in a non-uniform way in different regions of the momentum space. This gives rise to a breakup of the Fermi surface and to the formation of hot and cold regions, whose position depends on the hole or electron like nature of the carriers in the system.

PACS numbers: 71.10.Fd, 71.27.+a, 74.20.Mn, 79.60.-i
\end{abstract}

The mechanism of high-temperature superconductivity remains mysterious after nearly two decades of research. Among many theoretical proposals, a line of thought stresses the importance of the proximity to a Mott insulating state and of understanding the anomalous metallic state originating from doping this Mott insulator, a state which is not described by Fermi-liquid theory [1]. Along this path two very different approaches have been implemented: variational wavefunction studies [2] and slave boson approaches [3]. These methods allow to connect the Mott insulator to an anomalous normal state, interpreting the phenomenon of high temperature superconductivity as a direct consequence of the proximity to the Mott Metal-Insulator Transition (MIT). Their early successes include the prediction of the d-wave symmetry of the order parameter, the existence of a pseudogap close to half-filling and the dome-like shape of the critical temperature.

In this letter we investigate this issue using the recently developed Cellular Dynamical Mean-Field Theory (CDMFT) 4], applied to the two-dimensional Hubbard model on the square lattice. The CDMFT method reduces a quantum many body problem to a cluster of sites embedded in an effective medium described by a Weiss function. This quantity is determined self-consistently and can accommodate various broken symmetries, such as antiferromagnetism or $d$-wave superconductivity. In this work we consider a metallic phase which does not break any symmetry and follow its evolution as a function of parameters, such as the doping with respect to the Mott insulator. States with long-range order, including superconductivity and antiferromagnetism [5], will be discussed in a following paper. CDMFT offers the advantage of treating both the coherent and the incoherent excitations (quasiparticle peak and Hubbard bands) on the same footing, capturing the short-range physics of singlet formation on links, using self-energies which provide a dynamical (i.e. frequency dependent) general- ization of the condensates of the early slave boson mean field theory. The Hubbard model Hamiltonian is:

$H=-\sum_{i, j, \sigma} t_{i j}\left(c_{i, \sigma}^{\dagger} c_{j, \sigma}+\right.$ h.c. $)+U \sum_{i} n_{i \uparrow} n_{i \downarrow}-\mu \sum_{i} n_{i}$

where $c_{i, \sigma}\left(c_{i, \sigma}^{\dagger}\right)$ are destruction (creation) operators for electrons of spin $\sigma, n_{i \sigma}$ is the density of $\sigma$-spin electrons, $U$ is the on-site repulsion and $\mu$ the chemical potential which determines the electron density $n=$ $1 / N_{c} \sum_{i \sigma}\left\langle c_{i \sigma}^{\dagger} c_{i \sigma}\right\rangle, N_{c}$ is the total number of sites. The hopping amplitude $t_{i j}$ is chosen to be limited to nearestneighbors $t$ and to next-nearest-neighbors $t^{\prime}$, and a strong repulsion $U=16 t$ is assumed. In the following we will study the hole-doped system $(n<1)$ with $t^{\prime}=-0.3 t$, and the electron-doped system with $t^{\prime}=+0.3 t$ and $n<1$ (equivalent to the $n>1$ system upon a particle-hole transformation which reverses the sign of $t^{\prime}$ ). Our main results can be summarized as follows:

i) the normal state approaching the MIT exhibits the phenomenon of momentum space differentiation [6] , similar to the results of [7]. Different regions of momentum space behave very differently : hot (resp. cold) regions are characterized by a small (resp. large) quasiparticle residue $Z_{k}$ and a large (resp. small) inverse scattering rate $\operatorname{Im} \Sigma$. This effect gets most pronounced close to the MIT point.

ii) while the features described in i) are characteristic of proximity to the MIT, their detailed mechanism and the location in $k$-space of the cold and hot regions depend on the band parameter $t^{\prime} / t$. In particular we find that the sign of $t^{\prime} / t$ for hole-like [electron-like] doped cuprates corresponds to a location of the cold region in the $k$-space around $\left(\frac{\pi}{2}, \frac{\pi}{2}\right)[(0, \pi),(\pi, 0)]$. This finding agrees with experimental observations [6] and earlier theoretical studies $8,9,10,11,12]$. Our study is complementary, but consistent, with direct exact diagonalization studies or cluster perturbation theory on large clusters 11]. Introducing the CDMFT self-consistency condition allows for 
a continuous set of dopings and the high resolution in frequency needed to study the formation and destruction of quasiparticles, as demonstrated in single-site Dynamical Mean-Field Theory (DMFT) 13 and, with remarkable success, in tests in one dimension [14]. In this paper, we argue that the proximity of the MIT is central in this phenomenon and we investigate this connection in detail.

As mentioned above, in CDMFT we have to solve for a cluster of sites embedded in a self-consistent noninteracting bath. Our cluster is a $2 \times 2$ plaquette (Fig. 1), and the bath is truncated to 8 sites, in order to solve the impurity model by means of a variant of the Exact Diagonalization (ED) algorithm used in single-site DMFT 15]. For the Matsubara frequency calculations we use an effective inverse temperature [15], which we set equal to $\beta=128$ in units of the half bandwidth $4 t$. The computa-
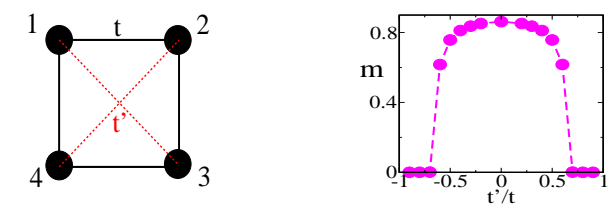

FIG. 1: Left side: the CDMFT Cluster. Right side: staggered magnetization as a function of the next-nearest hopping $t^{\prime}$ at half-filling. The parameter $t^{\prime}$ controls the magnetic frustration in the system.

tion produces the self-energies $\Sigma_{i j}$ on the plaquette and the lattice self-energy given by (using the square symmetry):

$$
\begin{array}{r}
\Sigma_{\text {latt }}(k, \omega)=\Sigma_{11}(\omega)+\Sigma_{12}(\omega)\left(\cos k_{x}+\cos k_{y}\right)+ \\
\Sigma_{13}(\omega) \cos k_{x} \cos k_{y}
\end{array}
$$

The cluster self-energies on the imaginary frequency axis (where the Green's functions of our ED discrete system are smooth [14]) are presented in Fig. 22 At large doping, we recover single-site DMFT results: $\Sigma_{12}, \Sigma_{13} \simeq 0$ and only $\Sigma_{11}$ appreciably different from zero. The lattice self-energy $\Sigma_{\text {latt }}(k, \omega)$ (1) is thereby $k$-independent. However the cluster self-energies increase sizably at low doping as we get close to the MIT and $\Sigma_{\text {latt }}$ becomes $k$-dependent. The zero frequency limit of the real part of the self-energy determines the shape of the interacting Fermi Surface (FS), which is given by $t_{\text {eff }}(k)=\mu$, where $t_{\text {eff }}(k) \equiv t(k)-\operatorname{Re} \Sigma_{\text {latt }}\left(k, \omega=0^{+}\right)$, being $t(k)$ the Fourier transform of the hopping amplitude $t_{i j}$. We find that the renormalization of the FS becomes appreciable close to the MIT. The self-energy itself depends weakly on the sign of $t^{\prime}$, and in particular it has the same sign for both positive and negative $t^{\prime}$. However, given its large magnitude, when combined with $t^{\prime}$ of different signs, it produces interacting FS's of very different shape in the electron-doped and hole-doped case (see Fig. 4). This can be understood in terms of the renormalized low energy hopping coefficients $t_{\text {eff }}=t-\operatorname{Re} \Sigma_{12}\left(\omega=0^{+}\right) / 2$

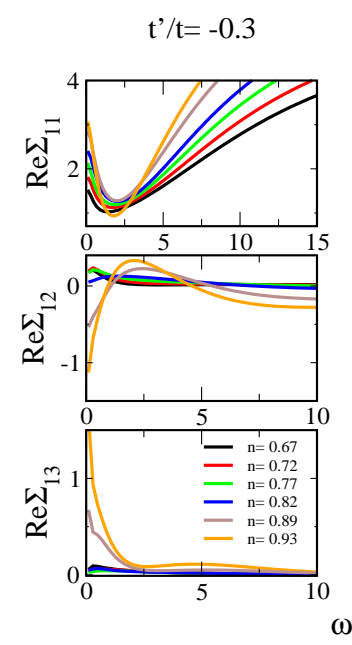

$t^{\prime} / t=+0.3$

$t^{\prime} / t=-0.3$

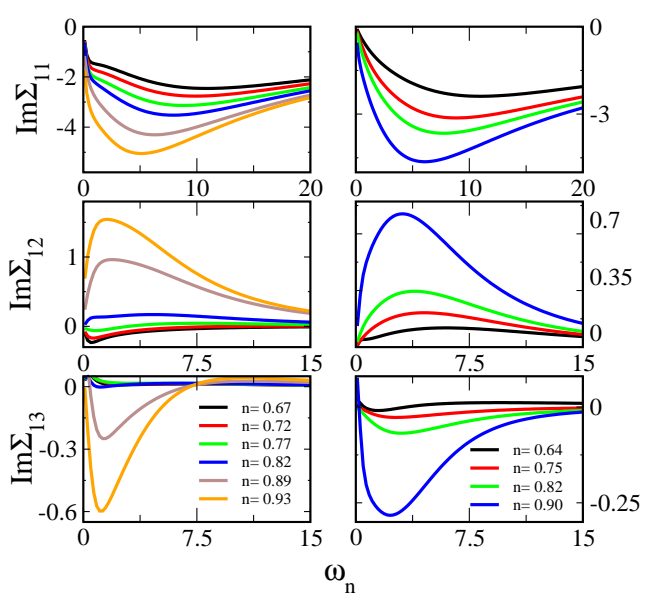

FIG. 2: Real and Imaginary part of the cluster self-energies for $t^{\prime} / t= \pm 0.3$. Notice the strong enhancement of the nearestneighbor an next-nearest-neighbor cluster self-energies $\Sigma_{12}$ and $\Sigma_{13}$ close to the MIT

and $t_{\text {eff }}^{\prime}=t^{\prime}-\operatorname{Re} \Sigma_{13}\left(\omega=0^{+}\right) / 4$ presented in Fig. 3 (where the $\omega=0^{+}$limit is extrapolated from the lowest Matsubara frequencies). Correlations act to increase the value of $t_{\text {eff. }} \operatorname{Re} \Sigma_{13}$ is negative, and it affects $t_{\text {eff }}^{\prime}$ quite differently for $t^{\prime} / t= \pm 0.3$. In fact it increases $t_{\text {eff }}^{\prime}$ in absolute value for $t^{\prime}=-0.3 t$, in such a way that also the ratio $\left(t_{\text {eff }}^{\prime} / t_{\text {eff }}\right) /\left(t^{\prime} / t\right)$ weakly increases approaching the MIT, thereby enhancing the hole-like curvature of the FS. This has the effect of flattening horizontally the Fermi line in the region of the momentum space close to $(0, \pi)$ or $(\pi, 0)$. Because of the enhancement in $t_{\mathrm{eff}}$ in the vicinity of the Mott transition point, in this region the Fermi line is also weakly doping dependent. This behavior of the antinodal region of cuprates superconductors in the normal state has been observed experimentally [16]. On the other hand, $\operatorname{Re} \Sigma_{13}$ decreases $t_{\text {eff }}^{\prime}$ for $t^{\prime}=+0.3 t$, driving the FS towards a flatter, nested FS as half-filling is approached. This is clearly seen in Fig. 4 where the spectral function at zero frequency is presented in the 

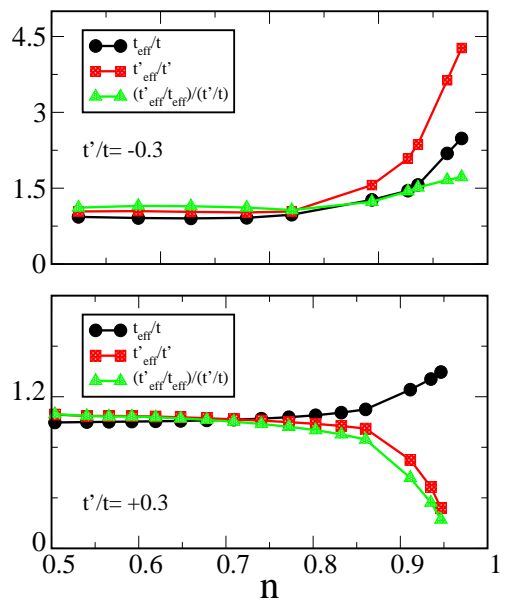

FIG. 3: Renormalization of the hopping coefficients and of their ratio as a function of density for $t^{\prime} / t= \pm 0.3$.
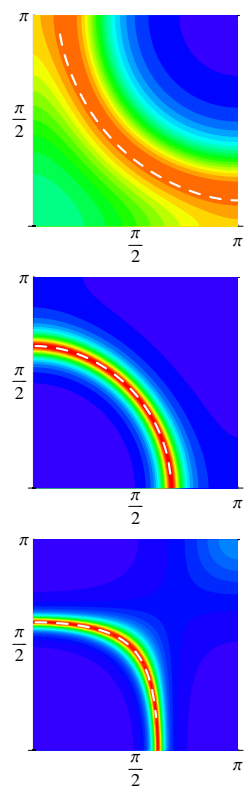

0.0

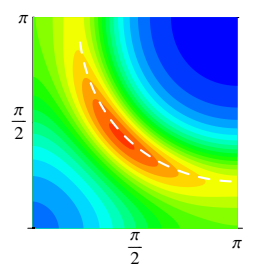

$\frac{\pi}{2}$
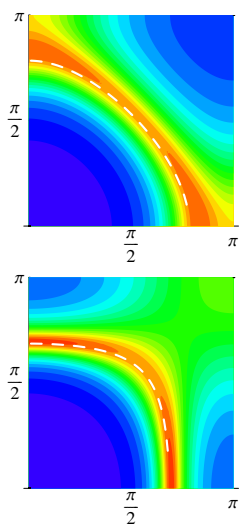
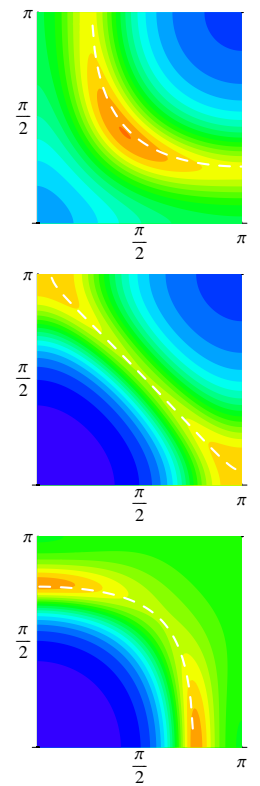

$x$
FIG. 4: $A\left(k, \omega=0^{+}\right)$in the first quadrant of the Brillouin zone. From the top: in the first row $t^{\prime}=-0.3 t$, $n=0.73,0.89,0.96$, color scale $x=0.28,0.22,0.12$; in the second $t^{\prime}=+0.3 t, n=0.70,0.90,0.95$, color scale $x=0.82,0.34,0.27$; in the lowest row $t^{\prime}=+0.9 t, n=$ $0.69,0.92,0.96$, color scale $x=0.90,0.32,0.22$. The white dashed line is the FS given by $t_{\mathrm{eff}}(k)=\mu$.

first quadrant of the Brillouin Zone.

Let us now turn to the formation of hot and cold regions. For both cases $t^{\prime} / t= \pm 0.3, \operatorname{Im} \Sigma_{11}$ and $\operatorname{Im} \Sigma_{13}$ have the same negative sign in contrast with the halffilled case of earlier studies on an anisotropic model [7]. While at $T=0, \operatorname{Im} \Sigma_{i j}(\omega=0)=0$, the finite effective temperature used in the ED calculation gives rise to lifetimes which are strongly dependent on the position on the FS and reflect the variation of the quasi-particle residue $Z_{k}=\left(1-\left.\frac{\partial \operatorname{Im} \Sigma_{\text {latt }}\left(k, i \omega_{n}\right)}{\partial \omega_{n}}\right|_{\omega_{n} \rightarrow 0}\right)^{-1}$. The slopes of all the imaginary parts of cluster self-energies increase as we approach the Mott transition (see Fig. 2). As they sum up differently to form $Z_{k}^{-1}$ in different regions of the $k$-space, according to the $k$-dependent coefficient multiplying them, $Z_{k}$ may assume different values on different

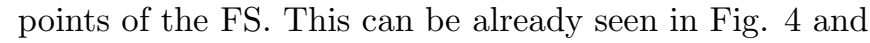
5

In the case $\mathbf{t}^{\prime}=+\mathbf{0 . 3} \mathbf{t}$ (electron-doped), as remarked above, the FS is flat and crosses $(\pi / 2, \pi / 2)$. There $\operatorname{Im} \Sigma_{\text {latt }}(k) \simeq \operatorname{Im} \Sigma_{11}$. On the other hand, near $(\pi, 0)$ $[(0, \pi)], \operatorname{Im} \Sigma_{\text {latt }}(k) \simeq \operatorname{Im} \Sigma_{11}-\operatorname{Im} \Sigma_{13}$. Since $\operatorname{Im} \Sigma_{11}$ and $\operatorname{Im} \Sigma_{13}$ have the same sign, we see that, while the local self-energy $\Sigma_{11}$ tends to decrease the quasiparticle residue and increase the inverse scattering rate while approaching the Mott transition, its effect is counterbalanced by the growth of $\operatorname{Im} \Sigma_{13}$, resulting in the formation of a cold patch around $(0, \pi)[(\pi, 0)]$. For $\mathbf{t}^{\prime}=-\mathbf{0 . 3 t}$ instead the effect of the real part of the self-energy is to bend the Fermi line slightly away from $(\pi / 2, \pi / 2)$. The intersection of the Fermi line and the zone diagonal now occurs at $(0.4 \pi, 0.4 \pi)$. At that point $\cos k_{x}+\cos k_{y} \simeq 0.6$, while $\cos k_{x} \cos k_{y}$ is numerically much smaller $(\sim 0.1)$. Hence $\operatorname{Im} \Sigma_{\text {latt }} \simeq \operatorname{Im} \Sigma_{11}+0.6 \operatorname{Im} \Sigma_{12}+0.1 \operatorname{Im} \Sigma_{13}$. The growth of $\operatorname{Im} \Sigma_{12}$ (which has the opposite sign to $\operatorname{Im} \Sigma_{11}$ and $\operatorname{Im} \Sigma_{13}$ ) reduces the strong correlation effects along the diagonal, and produces a cold patch. This "screening" of the correlations is larger than the one that takes place near the $(0, \pi)[(\pi, 0)]$ region, where the screening is given by $\operatorname{Im} \Sigma_{13}$. The modulation in $k$-space becomes in fact noticeable as soon as $\operatorname{Im} \Sigma_{12} \geq 1.7\left|\operatorname{Im} \Sigma_{13}\right|$. We argue that this effect is a precursor to the localization of particles that takes place at MIT. The hot quasiparticles localize, showing an insulator-like nature, while the cold quasiparticles retain their dispersion as the Mott insulator is approached. This can be seen also in Fig. 5] where we show the spectral weight $A(k, \omega)$ (at $4 \%$ doping for $t^{\prime} / t=-0.3,5 \%$ for $t^{\prime} / t=+0.3$ ) as a function of the energy for different points in the Brillouin zone, along the path $(0,0) \rightarrow(\pi, \pi) \rightarrow(0, \pi) \rightarrow(0,0)$. In the case $t^{\prime} / t=-0.3$ (the upper panel), a quasiparticle peak disperses along the $(0,0) \rightarrow(\pi, \pi)$, where a cold spot is formed at the Fermi level; on the contrary the peak is less dispersive passing through the region around $(0, \pi)$, where there is the hot spot. On the other hand, in the case $t^{\prime} / t=+0.3$ (lower panel) the quasiparticle peak is dispersing more in the vicinity of the region $(0, \pi)$ and, while dispersing less, evaporates around $(\pi / 2, \pi / 2)$. The hot-cold spots are in this way switched.

For $t^{\prime}=+0.3 t$ the Mott insulating state has antiferromagnetic long range order. To investigate the influence of antiferromagnetic correlation in determining the evolution of the electronic structure described above, we have 


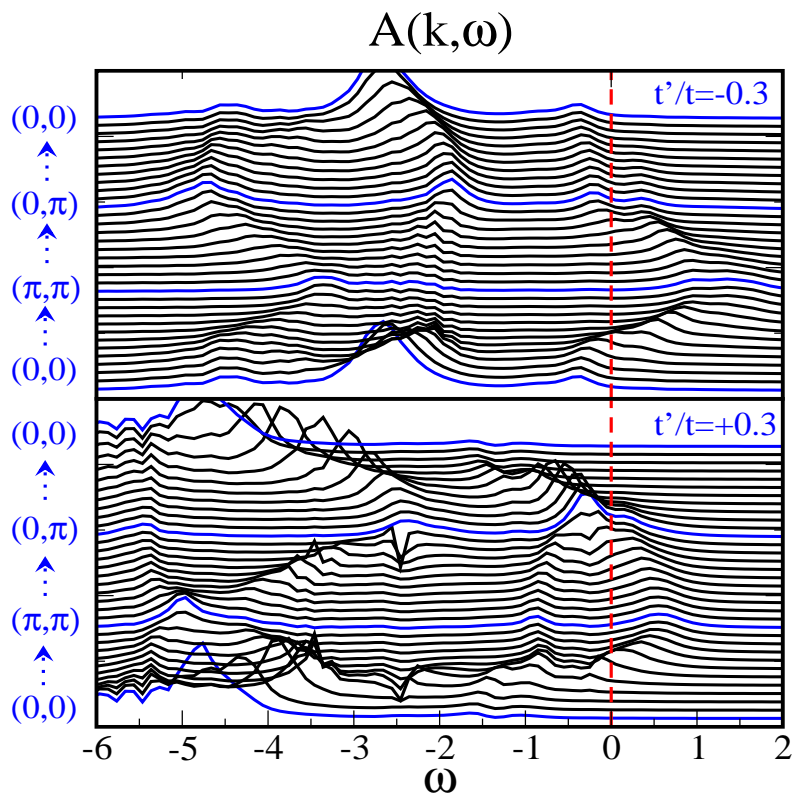

FIG. 5: Spectral function $A(k, \omega)$ as a function of $\omega$ along the path $(0,0) \rightarrow(\pi, \pi) \rightarrow(0, \pi) \rightarrow(\pi, \pi)$. In the upper panel $t^{\prime} / t=-0.3$ and $n=0.96$, in the lower panel $t^{\prime} / t=+0.3$ and $n=0.95$. The upper Hubbard band is out of the energy scale covered by the figure.

studied (see Fig. 3) the model with a large $t^{\prime}=+0.9 t$. For this value of $t^{\prime}$, in fact, we can rule out any antiferromagnetic long-range order already at half-filling. The right side of Fig. 1 displays the staggered magnetization of the system at half-filling, $m=\sum_{i}(-1)^{i}\left(n_{i \uparrow}-n_{i \downarrow}\right) / N_{c}$, as a function $t^{\prime} / t$, from $t^{\prime} / t=-0.9$ to $t^{\prime} / t=+0.9$ : a sudden drop of the magnetization is evident around $\left|t^{\prime} / t\right| \simeq 0.75$. The parent insulator state in this case is likely to have valence-bond or flux order 17. Despite the different nature of the parent insulator, the results for $t^{\prime}=+0.9 t$ are similar to those for $t^{\prime}=+0.3 t$. This, however, does not exclude that qualitative differences may appear between big and small $t^{\prime}$ with a better resolution in $k$-space (i.e. a bigger cluster).

To summarize, using Cellular DMFT we have studied the MIT in the Hubbard model. The approach to the insulator is not Fermi-liquid-like, and anisotropic properties appear in the momentum space. In particular, close to the MIT there is a breakdown of the FS and the appearance of cold and hot regions, whose precise location is the result of an interplay of the renormalization of the real and the imaginary parts of the self-energy. The emergence of these regions is a consequence of the proximity of the density-driven Mott transition. In the hole-doped case, the quasiparticles survive in the diagonal of the Brillouin zone, near $(\pi / 2, \pi / 2)$. This state, which has a fermionic spectrum with pointzeroes, is reminiscent of the flux phases and can be connected smoothly to the quasiparticles of the superconducting state. The electron-doped case is completely different. On a technical level, it is harder to approach the MIT closely, and a first-order phase transition may pre-empt a continuous approach to the insulating state. Furthermore, the FS is renormalized towards nesting, and the quasiparticles survive in a small region around $(\pi, 0)$ and $(0, \pi)$. These quasiparticles cannot be easily deformed into the superconducting state as opposed to the quasiparticles which live around $(\pi / 2, \pi / 2)$. These properties of the underlying normal state of the Hubbard model have striking resemblance to what is observed in the cuprates. The holedoped materials have a superconducting region which appears almost immediately after doping the Mott insulator. This superconducting state evolves continuously into the pseudogap state which in turn evolves continuously from the Mott insulator. In the electron-doped case, the pseudogap region, if it exists, is small, and a much larger doping is needed to reach the superconducting phase, which does not seem connected with the Mott insulator.

After the completion of this work, we became aware of a related work 18 on the $t^{\prime}=0$ case for the two dimensional Hubbard Model. We thank K.M. Shen and Z.-X. Shen for sharing their experimental results, A-M. Tremblay, D. Sénéchal, T. Stanescu, C. Castellani and A. Georges for useful discussions. Massimo Capone acknowledges Italian MIUR Cofin 2003 and the NSF support under grant DMR-0096462.

[1] P. W. Anderson, Science 2351196 (1987).

[2] C. Gros, R. Joint and T. M. Rice, Phys. Rev. B 36 381 (1997); A. Paramekanti, M. Randeria and N. Trivedi, Phys. Rev. Lett 87217002 (2001).

[3] G. Baskaran, Z. Zou and P. W. Anderson, Solid State Com. 63973 (1987); G. Kotliar and J. Liu, Phys. Rev. B 385142 (1988).

[4] G. Kotliar, S. Y. Savrasov, G. Palsson and G. Biroli, Phys. Rev. Lett 87186401 (2001).

[5] Th. Maier, M. Jarrell, Th. Pruschke and J. Keller, Phys. Rev. Lett 851524 (2000); A. I. Lichtenstein and M. I. Katsnelson, Phys. Rev. B 62 R9283 (2000).

[6] A. Damascelli, Z. X. Shen and Z. Hussain, Rev. Mod. Phys. 75473 (2003); "Physics of Superconductors II" J. C. Campuzano, M. R Norman and M. Randeria, K. H. Bennemann and J. B. Ketterson (2004) 167-273.

[7] O. Parcollet, G. Biroli and G. Kotliar, Phys. Rev. Lett 92226402 (2004).

[8] Th. A. Maier, Th. Pruschke and M. Jarrell, Phys. Rev. B 66075102 (2002).

[9] C. Kusko, R. S. Markiewicz, M. Lindroos and A. Bansil, Phys. Rev. B 66140513 (2002).

[10] B. Kyung, V. Hankevych, A.-M. Dare and A.-M.S. Tremblay, Phys. Rev. Lett 93147004 (2004).

[11] D. Sénéchal and A-M. S. Tremblay, Phys. Rev. Lett 92 126401 (2004).

[12] T. Tohyama and S. Maekawa, Phys. Rev. B 493596 
(1994).

[13] A. Georges, G. Kotliar, W. Krauth and M. J. Rozenberg, Rev. Mod. Phys. 6813 (1996).

[14] M. Capone, M. Civelli, S. S. Kancharla, C. Castellani and G. Kotliar, Phys. Rev. B 69195105 (2004).

[15] M. Caffarel and W. Krauth, Phys. Rev. Lett 721545
(1994).

[16] K. M. Shen et al., preprint and private communication..

[17] I. Affleck and B. Marston, Phys. Rev. B 373774 (1998); G. Kotliar, Phys. Rev. B 373664 (1998).

[18] B. Kyung et al., private communication. 